\title{
Influenza M1 Virus-Like Particles Consisting of Toxoplasma gondii Rhoptry Protein 4
}

\author{
Su-Hwa Lee', Dong-Hun Lee', Ying Piao², Eun-Kyung Moon³, Fu-Shi Quan ${ }^{3, *}$ \\ ${ }^{1}$ Department of Biomedical Science, Graduate School, Kyung Hee University, Seoul 02447, Korea; '2Department of Emergency, Yanbian University \\ Hospital, Yanji City, P. R. China; ${ }^{3}$ Department of Medical Zoology, Kyung Hee University School of Medicine, Seoul 02447, Korea
}

\begin{abstract}
Toxoplasma gondii infections occur throughout the world, and efforts are needed to develop various vaccine candidates expressing recombinant protein antigens. In this study, influenza matrix protein (M1) virus-like particles (VLPs) consisting of $T$. gondii rhoptry antigen 4 (ROP4 protein) were generated using baculovirus (rBV) expression system. Recombinant ROP4 protein with influenza M1 were cloned and expressed in rBV. SF9 insect cells were coinfected with recombinant rBVs expressing T. gondii ROP4 and influenza M1. As the results, influenza M1 VLPs showed spherical shapes, and T. gondii ROP4 protein exhibited as spikes on VLP surface under transmission electron microscopy (TEM). The M1 VLPs resemble virions in morphology and size. We found that M1 VLPs reacted with antibody from T. gondii-infected mice by western blot and ELISA. This study demonstrated that $T$. gondii ROP4 protein can be expressed on the surface of influenza M1 VLPs and the M1 VLPs containing T. gondii ROP4 reacted with T. gondii-infected sera, indicating the possibility that M1 VLPs could be used as a coating antigen for diagnostic and/or vaccine candidate against $T$. gondii infection.
\end{abstract}

Key words: Toxoplasma gondii, cloning, influenza matrix protein, baculovirus, virus-like particle, antibody

\section{INTRODUCTION}

Toxoplasma gondii is a protozoan parasite that can infect most animals and humans, with a worldwide distribution, permanently infecting nearly $20 \%$ of the global population [14]. Specific groups of patients who are immunologically impaired could be severe. Thus, prevention and diagnosis of $T$. gondii infection become crucial for the surveillance and control. Recent important progress has been made identifying anti-toxoplasma vaccine candidates that can stimulate an immunological response [4]. However, vaccine efficacy is not successful. The diagnosis of toxoplasmosis in humans is made by biological, serological, histological, molecular methods, parasite isolation, or by some combination of the above $[5,6]$. Tachyzoite lysate antigen (TLA) as a coating antigen used in conventional indirect ELISAs showed different results, resulting in difficulty to standardize and evaluation. Commercial test kits were used to determine IgM and/or IgG antibodies

- Received 28 December 2016, revised 2 March 2017, accepted 11 March 2017.

*Corresponding authors (fsquan@khu.ac.kr)

(c) 2017, Korean Society for Parasitology and Tropical Medicine

This is an Open Access article distributed under the terms of the Creative Commons Attribution Non-Commercial License (http://creativecommons.org/licenses/by-nc/4.0) which permits unrestricted non-commercial use, distribution, and reproduction in any medium, provided the original work is properly cited. showed false-positive, and the reported results are difficult to interpret [6-8]. Thus, recombinant proteins as alternative approach have been used since the recombinant protein showed advantages in the precision and standardization of the antigen [6].

Numerous recombinant antigens, including granule antigens GRA1, GRA2, GRA4, GRA6, GRA7, and GRA8, rhoptry proteins ROP1 and ROP2, matrix protein MAG1, microneme proteins MIC2, MIC3, MIC4, and MIC5, and surface antigens SAG1 and SAG2, have been expressed in Escherichia coli or yeast, and their potential diagnostic value or vaccine efficacy were evaluated in humans or animals [9-14]. We recently reported that recombinant virus-like particles (VLPs), containing T. gondii inner membrane complex (IMC), have shown that VLPs are highly immunogenic [4]. Since VLPs are formed on the surface with high-density particles acting as antigens, which can induce a high immune response [15], indicating that VLPs could be used as a protein antigen for diagnostic potential or vaccine candidate.

Malaria is one of the most common infectious diseases and a great public health problem worldwide. Identification of potential diagnostic and vaccine development are particularly important. Since rhoptry protein in malaria parasite is very important in invasion of its host cells [16], and rhoptries are 
major players in T. gondii invasion also [17], we assume that cross-reactivity may exist between $T$. gondii and malaria. In this study, we, for the first time, generated virus-like particles containing $T$. gondii ROP4 protein. We found that T. gondii ROP4 protein can be expressed on the surface of influenza M1 VLPs. VLP protein antigens showed IgG reactivity with $T$. gondii-infected sera, and IgG cross-reactivity with Plasmodium berghei malaria-infected sera.

\section{MATERIALS AND METHODS}

\section{Parasites, cells, and antibodies}

Toxoplasma gondii RH strain and ME49 strain were maintained according to the methods described previously [18-20]. T. gondii RH stain was used for RNA extraction, and T. gondii ME49 was used to infect mice and to collect sera. Spodoptera frugiperda Sf9 cells were maintained in suspension in serum-free SF900 II medium (Invitrogen, Carlsbad, California, USA) at $27^{\circ} \mathrm{C}$ in spinner flasks at 130 to $140 \mathrm{rpm}$. S. frugiperda Sf9 cells were used for production of recommended baculovirus (rBV) and virus-like particles. Horseradish peroxidase (HRP)-conjugated goat antimouse immunoglobulin $\mathrm{G}(\mathrm{IgG})$ was purchased from Southern Biotech (Birmingham, Alabama, USA).

\section{Cloning of $T$. gondii rhoptry protein (ROP4) and influenza M1}

T. gondii RH strain was collected from mice, and RNA was extracted using RNeasy Mini Kit (Qiagen, Valencia, California, USA). Total RNA was reversely transcribed to CDNA using Prime Script 1st strand cDNA synthesis kit according to the manufacturer's instructions (Takara, Otsu, Japan). T. gondii ROP4 gene was amplified by PCR from cDNA with primers. The primers were designed according to the nucleotide sequence of ROP4 in GenBank (accession no. EU047558): forward (5'-AAAGCATGCACCATGGGGCACCCTACCTCTTT-3') and reverse (5'-TTAGGTACCTCACGTTTCCGGTGGTGGCAT-3') with SphI and KpnI restriction enzyme sites (underlined). PCR product was cloned into pFastBac vector (Invitrogen) as described previously [21]. For influenza M1 (accession no. EF4 67824, 1,027 bp) gene cloning, A/PR/8/34 virus was inoculated into MDCK cells, and total viral RNA was extracted as mentioned above. Reverse transcription (RT) and PCR were performed on extracted viral RNA using the 1-step RT-PCR system (Invitrogen) with gene specific oligonucleotide primers. The following primer pairs were used for M1: 5'-TCCCCCGGG
CCACCATGAGCCTTCTGACCGAGGTC-3'; reverse primer, 5'-TTACTTCTAGATTACTTGAACCGTTGCATCTG-3'; SmaI and $\mathrm{X} b a \mathrm{I}$ sites are underlined. A cDNA fragment containing the M1 was cloned into pFastBac vector (Invitrogen). The recombinant plasmids ROP4-pFastBac or M1-pFastBac were transformed into an E. coli DH5- $\alpha$. The targeted fragments of the ROP4 gene and M1 gene were identified by restriction digestion and sequencing analysis. Confirmed recombinant plasmids were transformed into a DH10-Bac and extracted using FavorPrep gel purification Kit (Favorgen, Cheshire, UK). The recombinant plasmid DNAs (DH10-Bac) were stored at $-20^{\circ} \mathrm{C}$ until used.

\section{Generation of recombinant baculovirus (rBV) and VLPS}

To generate $\mathrm{rBV}$, transfections of recombinant plasmid ROP4-pFastBac or M1-pFastBac were transfected into the Sf9 cells using cellfectin II (Invitrogen) as according to the manufacturer. To produce VLPs containing ROP4 and M1, Sf9 cells were coinfected with rBVs expressing ROP4 or M1. VLPs released into the cell culture supernatants were harvested 3 days after infection and cleared by centrifugation at 6,000 rpm for $30 \mathrm{~min}$ at $4^{\circ} \mathrm{C}$ to remove cells. Supernatants containing VLPs were concentrated by high-speed centrifugation $(45,000 \mathrm{~g}$ for $30 \mathrm{~min}$ ) and purified through a $15-30-60 \%$ discontinuous sucrose gradient at $45,000 \mathrm{~g}$ for $1 \mathrm{hr}$ at $4{ }^{\circ} \mathrm{C}$. VLP bands between $30 \%$ and $60 \%$ were harvested and pelleted by high-speed centrifugation (45,000 g for $30 \mathrm{~min}$ ). The VLPs were resuspended in $0.1 \mathrm{M} \mathrm{PBS}$ overnight at $4^{\circ} \mathrm{C}$ and concentration was determined using QuantiPro BCA Assay Kit (Sigma-Aldrich, St. Louis, Missouri, USA).

\section{Characterization of VLPS}

To characterize the VLPs, the morphology was confirmed by electron microscopy. For electron microscopy, negative staining of VLPs was performed followed by transmission electron microscopy as described previously (Tecnai G2 spirit, FEI, Hillsboro, Oregon, USA) [21].

\section{Reactivity of VLPs with T. gondii-infected mouse sera}

Female inbred BABL/c (aged 8 weeks; Nara Biotech, Kyonggi-do, Korea) were used. All animal experiments and husbandry involved in these studies were conducted under the guidelines of the Kyung Hee University IACUC. Mice were infected with T. gondii (ME49), and the mouse sera were collected at week 4 and stored at $-20^{\circ} \mathrm{C}$ until used. VLPs containing T. gon- 
dii ROP4 protein was identified by western blot. Monoclonal mouse anti-M1 antibody was used to confirm influenza M1 protein content. The levels of $\operatorname{IgG}$ antibody was determined by ELISA. Flat-immunoplates (96-well) were coated with $100 \mu \mathrm{l}$ of VLPs at a concentration of $4 \mu \mathrm{g} / \mathrm{ml}$ in $0.05 \mathrm{M}$ carbonate bicarbonate buffer ( $\mathrm{pH}$ 9.6) per well and incubated overnight at $4^{\circ} \mathrm{C}$. The mouse serum samples were serially diluted in PBST (100 $\mathrm{\mu l} /$ well) and incubated in the plates for $1.5 \mathrm{hr}$ at $37^{\circ} \mathrm{C}$. Horseradish peroxidase (HRP)-conjugated goat anti-mouse immunoglobulin $\mathrm{G}(\mathrm{IgG})$ was purchased from Southern Biotech.

\section{IgG cross-activity of VLPs with Plasmodium berghei antibody}

IgG cross-reactivity of VLPs was determined by reacting with Plasmodium berghei-infected mouse sera. P. berghei was kindly provided by Dr. YC Hong at Kyungpook National University. Mice were infected with $P$. berghei, and mouse sera were collected at 4 weeks and stored at $-20^{\circ} \mathrm{C}$ until used. ELISA was performed to determine IgG cross-reactivity between T. gondii and P. berghei. T. gondii-, and P. berghei-infected mouse sera
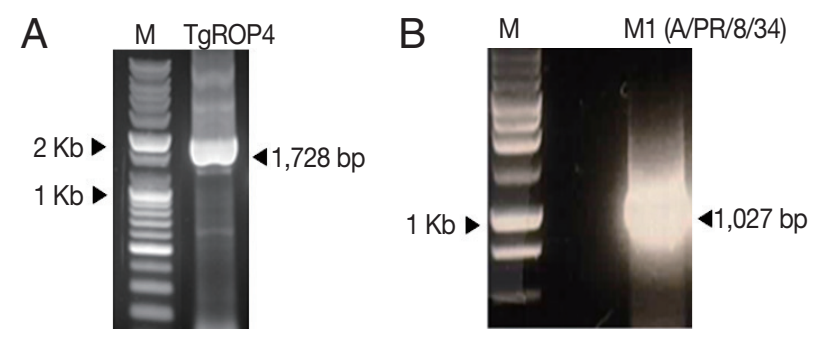

Fig. 1. PCR amplification of T. gondii ROP4 (A) and influenza M1 genes (B). T. gondii ROP4 (1,728 bp) gene was RCR-amplified from cDNA synthesized using a Prime Script 1st Strain cDNA Synthesis Kit using total RNA extracted from T. gondii RH. Influenza M1 gene was PCR amplified from total RNA extract from influenza virus (A/PR/8/34). M, DNA marker; TgROP4, T. gondii ROP4; M1, influenza M1.
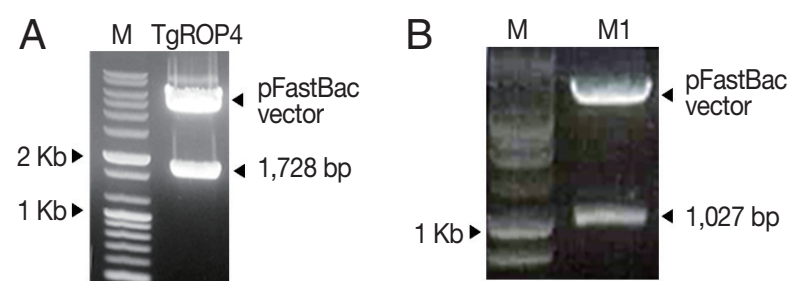

Fig. 2. Construction of pFastBac vectors. T. gondii ROP4 gene and influenza M1 were cloned into the pFastBac with Sphl/Kpnl and EcoRI/Xhol enzymes, respectively, resulting in pFastbac plasmids containing T. gondii ROP 4 (A) or M1 (B). were used for primary antibodies at 1:50 dilution, and HRPconjugated goat anti-mouse IgG antibodies were used as secondary antibodies.

\section{Statistics}

All parameters were recorded for individuals within groups. Data were compared using analysis of variance and the nonparametric 1-way Kruskal-Wallis test in the PC-SAS system (SAS Institute, Cary, North Carolina, USA). A P-value of $<0.05$ was considered to be significant.

\section{RESULTS}

\section{Recombinant constructs generated}

T. gondii ROP4 and influenza M1 genes were amplified by PCR or RT-PCR, respectively. As shown in Fig. 1, ROP4 was 1,728 bp (A), and M1 was 1,027 bp (B) in size, respectively. The amplified PCR products were cloned into pFastBac vectors, and the insertions of ROP4 and M1 in pFastBac vectors were confirmed by restriction enzymes (Fig. 2A, B). The nucleotide sequences of the $\mathrm{T}$. gondii ROP4 and influenza M1 genes were identical to previously published sequences (accession nos. EU047558 for T. gondii ROP4 and EF467824 for M1) by DNA sequencing (Eurofins MWG Operon).

\section{Influenza M1 VLPs produced}

To produce M1 VLPs, recombinant baculovirus (rBV) expressing T. gondii ROP4 or influenza M1 were co-infected into Sf9 cells, and thus, M1 VLPs were produced. The VLP-producing Sf9 cells (Fig. 3B) are significantly larger in size than normal control Sf9 cells (Fig. 3A). The size and morphology of in-
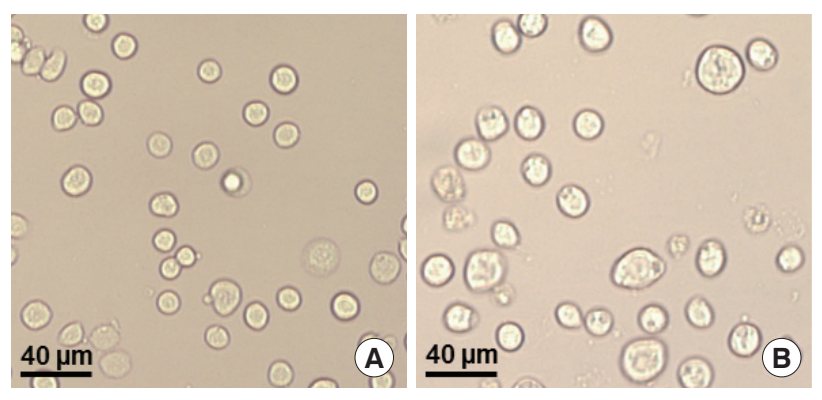

Fig. 3. Production of virus-like particles (VLPS). pFastBac plasmids containing ROP4 or M1 were transfected into Sf9 cells, respectively, and baculoviruses expressing T. gondii ROP4 or influenza M1 were generated. Recombinant baculovirus was coinfected into Sf9 cells, and the VLPs were produced. A, Normal SF9 cells; B, VLP-producing cells. 


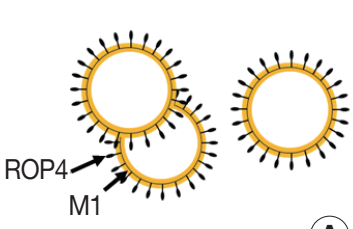

(A)

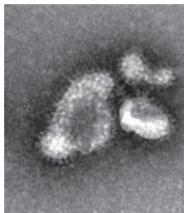

$100 \mathrm{~nm}$
B

Fig. 4. Electron microscopy examination. Transmission electronic microscopy was used to characterize M1 VLPs morphologically. Negative staining electron microscopy of influenza M1 VLPS was performed. A, Diagram of the M1 VLPS; B, M1 VLPs containing T. gondii ROP 4 under EM.

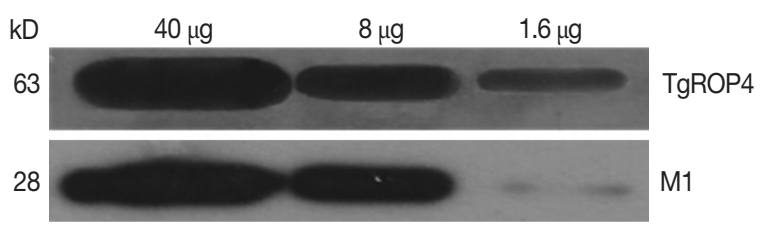

Fig. 5. Reactivity of M1 VLPS with T. gondii antibody by western blot. A total of 40,8 , and $1.6 \mu \mathrm{g}$ of M1 VLPs were loaded per lane. Polyclonal mouse anti-T. gondii (ME49) antibody was used to probe T. gondii ROP4 protein (63kD), and monoclonal anti-M1 antibody was used to probe influenza M1 protein (28kD). Thus, T. gondii ROP4 (TgROP4) and influenza M1 proteins were identified, respectively, by western blot.

fluenza VLPs were examined under electron microscopy. M1 VLPs showed spherical shapes with spikes on their surfaces, and generated M1 VLPs resembled virions in morphology and size (Fig. 4A, B).

\section{Influenza M1 VLPs reacted with sera from T. gondii (ME49)-infected mice}

Influenza M1 VLPs containing ROP4 were used to react with antibodies from T. gondii-infected mice by western and ELISA. As seen in Fig. 5, the incorporation of T. gondii ROP4 (63 kDa) and influenza M1 (28 kDa) into VLPs was confirmed by western blot. As seen in Fig. 6, compared to naïve control mouse sera, high levels of IgG antibodies were detected from T. gondiiinfected mouse sera at 1:100, 1:500, and 1:2,500 dilutions when M1 VLPs were used as coating antigens by ELISA.

\section{Influenza M1 VLPs induced IgG cross-reactivity with Plasmodium berghei-infected sera}

Influenza M1 VLPs containing T. gondii ROP4 were used to determine IgG cross-reactivity with antibodies from $P$. bergheiinfected mice. As seen in Fig. 7, compared to naïve mouse control, higher levels of IgG antibodies were detected from $P$. berghei-infected mouse sera, indicating IgG cross-reactivity be-

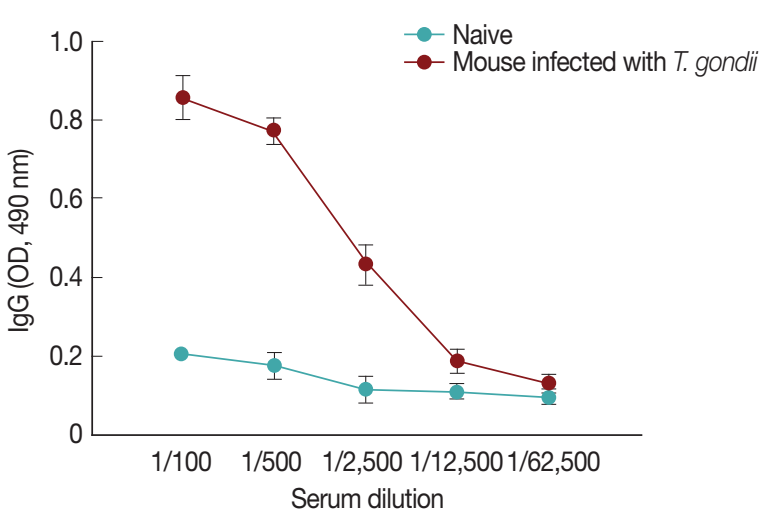

Fig. 6. M1 VLPs as a coating antigen reacted with $T$. gondii antibody. Mice were infected with T. gondii (ME49) and 1 month later, mouse sera were collected. M1 VLPs were coated onto 96-well plates, and collected sera were serially diluted and used as a primary antibody to determine lgG antibody responses using ELISA. Higher levels of lgG antibody responses were detected when M1 VLPS as coating antigens.

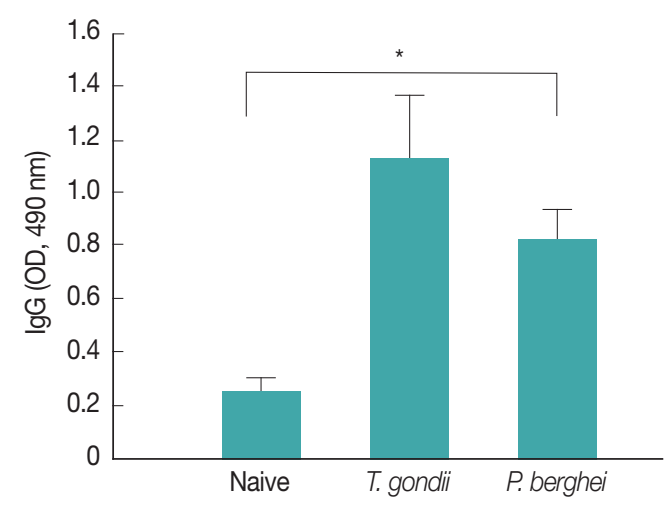

Fig. 7. IgG cross-reactivity of M1 VLPs containing T. gondii ROP4 with Plasmodium berghei-infected mouse sera. M1 VLPs containing T. gondii ROP4 were coated onto 96-well pates, and $P$. berghei-infected mouse sera were used as primary antibodies. Then, IgG cross-reactivity was determined using ELISA. A higher level of IgG cross-reactivity of M1 VLPs with $P$. berghei-infected sera was detected when M1 VLPs as coating antigens $\left({ }^{\star} P<\right.$ 0.05).

tween T. gondii and P. berghei.

\section{DISCUSSION}

In this study, for the first time, we successfully cloned $T$. gondii ROP4 gene into pFastBac vector and generated baculovirus expressing T. gondii ROP4. Influenza M1 protein expressed by baculovirus expression system contributed to the formation of influenza-like shape of VLPs [22]. During the VLP generation, baculoviruses expressing T. gondii ROP4 or M1 incorporated 
into VLPs, which showed spherical shapes (M1) with spikes (T. gondii ROP4) on the VLP surface [4]. We found that VLP proteins were clearly recognized by polyclonal antibodies of $T$. gondii by western blot (Fig. 5). VLPs reacted with antibodies from mouse infected with $T$. gondii (Fig. 6), indicating VLPs consisting of $T$. gondii ROP4, could be used as a coating antigen for diagnostic and/or vaccine candidate against $T$. gondii infection. To determine the cross-reactivity between $T$. gondii and malaria, in our current study, malaria mouse model $P$. berghei was used. VLPs containing T. gondii rhoptry structure showed IgG cross-reactivity with $P$. berghei-infected mouse sera (Fig. 7), indicating that VLPs could be used as a vaccine candidate against $P$. berghei infection as well. Further studies are needed to elucidate their potential as a vaccine candidate against T. gondii and other coccidian species infections having rhoptry structures.

Although, a number of different recombinant proteins of $T$. gondii expressed by $E$. coli have been used as antigens for diagnostic tool or vaccine candidates, the vaccine efficacy induced by the recombinant proteins expressed by E. coli is largely limited. Mice immunized with the recombinant protein vaccines mostly showed little protection against challenge infection [23-26]. In our recent study, we have reported that VLPs targeted T. gondii inner membrane complex sub-compartment (IMC) induced humoral and cellular immunity, resulting in protection (100\% survival) [4]. This promising result indicated that VLPs could be an alternative strategy for the novel vaccine [4]. The VLPs contained repetitive high density displays of T. gondii IMC surface proteins induced systemic and mucosal immune responses [15].

ROP4, a member of the prominent ROP2-protein family, is released from rhoptries, participating in the parasite-host cell penetration process [27]. Additionally, ROP4 antigen expressed by E. coli elicits strong cellular and humoral immune responses in immunized mice, which are partly protective against T. gondii challenge [28]. This T. gondii ROP4 protein is also useful as a diagnostic tool for a serological test [29]. Thus, in this study, we, for the first time, successfully produced VLPs containing $T$. gondii ROP4 protein in which influenza matrix $\mathrm{M} 1$ is as a core protein. For further studies, the protective immunity induced by VLPs immunization is needed as well as the evaluation of VLPs as a diagnostic tool for a serological test.

\section{ACKNOWLEDGMENTS}

This work was supported by a grant from the National Research Foundation of Korea (NRF) (no. NRF-2014R1A2A2A01 004899), a grant from the Agri-Bio Industry Technology Development Program (no. 315030-03-1-HD020), IPET, MAFRA, KHIDI, and a grant from the Ministry of Health and Welfare, Republic of Korea (no. HI15C2928).

\section{CONFLICT OF INTEREST}

The authors have no conflicts of interest to declare.

\section{REFERENCES}

1. Dubey JP. Toxoplasmosis of Animals and Humans. 2nd ed. Beltsville, Maryland, USA. CRC. 2010.

2. Robert-Gangneux F, Darde ML. Epidemiology of and diagnostic strategies for toxoplasmosis. Clin Microbiol Rev 2012; 25: 264296.

3. Chen J, Xu MJ, Zhou DH, Song HQ, Wang CR, Zhu XQ. Canine and feline parasitic zoonoses in China. Parasit Vectors 2012; 5: 152.

4. Lee DH, Lee SH, Kim AR, Quan FS. Virus-Like Nanoparticle Vaccine Confers Protection against Toxoplasma gondii. PLoS One 2016; 11: e0161231.

5. Hill D, Dubey J. Toxoplasma gondii: transmission, diagnosis and prevention. Clin Microbiol Infect 2002; 8: 634-640.

6. Liu Q, Wang ZD, Huang SY, Zhu XQ. Diagnosis of toxoplasmosis and typing of Toxoplasma gondii. Parasit Vectors 2015; 8: 292.

7. Liesenfeld O, Press C, Montoya JG, Gill R, Isaac-Renton JL, Hedman K, Remington JS. False-positive results in immunoglobulin M (IgM) toxoplasma antibody tests and importance of confirmatory testing: the Platelia Toxo IgM test. J Clin Microbiol 1997; 35: $174-178$.

8. Wilson M, Remington JS, Clavet C, Varney G, Press C, Ware D. Evaluation of six commercial kits for detection of human immunoglobulin $\mathrm{M}$ antibodies to Toxoplasma gondii. The FDA Toxoplasmosis Ad Hoc Working Group. J Clin Microbiol 1997; 35: 3112-3115.

9. Kotresha D, Noordin R. Recombinant proteins in the diagnosis of toxoplasmosis. APMIS 2010; 118: 529-542.

10. Lau YL, Fong MY, Mohd Idris M, Ching XT. Cloning and expression of Toxoplasma gondii dense granule antigen 2 (GRA2) gene by Pichia pastoris. Southeast Asian J Trop Med Public Health 2012; 43: 10.

11. Thiruvengadam G, Init I, Fong MY, Lau, YL. Optimization of the expression of surface antigen SAG1/2 of Toxoplasma gondii in the yeast Pichia pastoris. Trop Biomed 2011; 28: 506-513.

12. Chang PY, Fong MY, Nissapatorn V, Lau YL. Evaluation of Pichia 
pastoris-expressed recombinant rhoptry protein 2 of Toxoplasma gondii for its application in diagnosis of toxoplasmosis. Am J Trop Med Hyg 2011; 85: 485-489.

13. Wang Z, Ge W, Huang SY, Li J, Zhu XQ, Liu Q. Evaluation of recombinant granule antigens GRA1 and GRA7 for serodiagnosis of Toxoplasma gondii infection in dogs. BMC Vet Res 2014; 10 : 158.

14. Wang Z, Ge W, Li J, Song M, Sun H, Wei F, Liu Q. Production and evaluation of recombinant granule antigen protein GRA7 for serodiagnosis of Toxoplasma gondii infection in cattle. Foodborne Pathog Dis 2014; 11: 734-739.

15. Lee DH, Kim AR, Lee SH, Quan FS. Cross-protection induced by Toxoplasma gondii virus-like particle vaccine upon intraperitoneal route challenge. Acta Trop 2016; 164: 77-83.

16. Counihan NA, Kalanon M, Coppel RL, de Koning-Ward TF. Plasmodium rhoptry proteins: why order is important. Trends Parasitol 2013; 29: 228-236.

17. Dubremetz JF. Rhoptries are major players in Toxoplasma gondii invasion and host cell interaction. Cell Microbiol 2007; 9: 841848.

18. Wang PY, Yuan ZG, Petersen E, Li J, Zhang XX, Li XZ, Li HX, Lv ZC, Cheng T, Ren D, Yang GL, Lin RQ, Zhu XQ. Protective efficacy of a Toxoplasma gondii rhoptry protein 13 plasmid DNA vaccine in mice. Clin Vaccine Immunol 2012; 19: 1916-1920.

19. Joyce BR, Queener SF, Wek RC, Sullivan WJ Jr. Phosphorylation of eukaryotic initiation factor-2a promotes the extracellular survival of obligate intracellular parasite Toxoplasma gondii. Proc Natl Acad Sci USA 2010; 107: 17200-17205.

20. Chen J, Huang S, Li Z, Yuan Z, Zhou D, Petersen E, Zhang N, Zhu X. Protective immunity induced by a DNA vaccine expressing eIF4A of Toxoplasma gondii against acute toxoplasmosis in mice. Vaccine 2013; 31: 1734-1739.

21. Quan FS, Huang C, Compans RW, Kang SM. Virus-like particle vaccine induces protective immunity against homologous and heterologous strains of influenza virus. J Virol 2007; 81: 35143524.

22. Quan FS, Kim YH, Lee SJ, Yi H, Kang SM, Bozja J, Moore ML, Compans RW. Viruslike particle vaccine induces protection against respiratory syncytial virus infection in mice. J Infect Dis 2011; 204: 987-995.

23. Letscher-Bru V, Pfaff AW, Abou-Bacar A, Filisetti D, Antoni E, Villard O, Klein JP, Candolfi E. Vaccination with Toxoplasma gondii SAG-1 protein is protective against congenital toxoplasmosis in BALB/c mice but not in CBA/J mice. Infect Immun 2003; 71: 6615-6619.

24. Dlugonska H. Toxoplasma rhoptries: unique secretory organelles and source of promising vaccine proteins for immunoprevention of toxoplasmosis. J Biomed Biotechnol 2008; e632424

25. Holec-Gąsior, L. Toxoplasma gondii recombinant antigens as tools for serodiagnosis of human toxoplasmosis: current status of studies. Clin Vaccine Immunol 2013; 20: 1343-1351.

26. Jongert E, Roberts CW, Gargano N, Förster-Waldl E, Petersen E. Vaccines against Toxoplasma gondii: challenges and opportunities. Mem Inst Oswaldo Cruz 2009; 104: 252-266.

27. Dziadek B, Gatkowska J, Grzybowski M, Dziadek J, Dzitko K, Dlugonska H. Toxoplasma gondii: the vaccine potential of three trivalent antigen-cocktails composed of recombinant ROP2, ROP4, GRA4 and SAG1 proteins against chronic toxoplasmosis in BALB/c mice. Exp Parasitol 2012; 131: 133-138.

28. Dziadek B, Gatkowska J, Brzostek A, Dziadek J, Dzitko K, Dlugonska H. Toxoplasma gondii: the immunogenic and protective efficacy of recombinant ROP2 and ROP4 rhoptry proteins in murine experimental toxoplasmosis. Exp Parasitol 2009; 123: 81-89.

29. Gatkowska J, Dziadek B, Brzostek A, Dziadek J, Dzitko K, Dlugonska H. Determination of diagnostic value of Toxoplasma gondii recombinant ROP2 and ROP4 antigens in mouse experimental model. Pol J Microbiol 2010; 59: 137-141. 